\title{
Arm Position Constraints During Pointing and Reaching in 3-D Space
}

\author{
C.C.A.M. GIELEN,${ }^{1}$ E. J. VRIJENHOEK, ${ }^{1}$ T. FLASH,${ }^{2}$ AND S.F.W. NEGGERS ${ }^{1}$ \\ ${ }^{1}$ Department of Medical Physics and Biophysics, University of Nijmegen, NL 6525 EZ Nijmegen, The Netherlands; and \\ ${ }^{2}$ Department of Applied Mathematics and Computer Science, Weizmann Institute of Science, Rehovot 76100, Israel
}

Gielen, C.C.A.M., E. J. Vrijenhoek, T. Flash, and S.F.W. Neggers. Arm position constraints during pointing and reaching in 3D space. J. Neurophysiol. 78: 660-673, 1997. Arm movements in 3 -D space were studied to investigate the reduction in the number of rotational degrees of freedom in the shoulder and elbow during pointing movements with the fully extended arm and during pointing movements to targets in various directions and at various distances relative to the shoulder, requiring flexion/extension in the elbow. The postures of both the upper arm and forearm can be described by rotation vectors, which represent these postures as a rotation from a reference position to the current position. The rotation vectors describing the posture of the upper arm and forearm were found to lie in a 2-D (curved) surface both for pointing with the fully extended arm and for pointing with elbow flexion. This result generalizes on previous results on the reduction of the number of degrees of freedom from three to two in the shoulder for the fully extended arm to a similar reduction in the number of degrees of freedom for the upper arm and forearm for normal arm movements involving also elbow flexion and extension. The orientation of the 2-D surface fitted to the rotation vectors describing the position of the upper arm and forearm was the same for pointing with the extended arm and for movements with flexion/ extension of the elbow. The scatter in torsion of the rotation vectors describing the position of the upper arm and forearm relative to the $2-\mathrm{D}$ surface was typically $3-4^{\circ}$, which is small considering the range of $\sim 180$ and $360^{\circ}$ for torsional rotations of the upper arm and the forearm, respectively. Donders' law states that arm posture for pointing to a target does not depend on previous positions of the arm. The results of our experiments demonstrate that the upper arm violates Donders' law. However, the variations in torsion of the upper arm are small, typically a few degrees. These deviations from Donders' law have been overlooked in previous studies, presumably because the variations are relatively small. These variations may explain the larger scatter of the rotation vectors for arm movements $\left(3-4^{\circ}\right)$ than reported for the eye $\left(1^{\circ}\right)$. Unlike for saccadic eye movements, joint rotations in the shoulder during aiming movements were not all single-axis rotations. On the contrary, the direction of the angular velocity vector varied during the movement in a consistent and reproducible way, depending on amplitude, direction, and starting position of the movement. These results reveal several differences between arm movements during pointing and saccadic eye movements. The implications for our understanding of the coordination of eye and arm movements and for the planning of 3-D arm movements are discussed.

\section{INTRODUCTION}

It is well known that the orientation of an object after rotation along two noncolinear axes depends on the order of the rotations (Donders 1847; von Helmholtz 1925; Tweed and Vilis 1987). This phenomenon has severe implications for joints with three degrees of freedom, because it implies that the orientation of a limb will depend on previous joint rotations if no additional constraints on the rotations are imposed. Even worse, torsion of the shoulder or eye may accumulate to unphysiological values if no attention is paid to the rotations in the shoulder or the eye.

Yet, it is well known that the orientation of the eyes in the head is unique for each gaze direction (Donders 1847; Nakayama and Balliet 1977; Tweed and Vilis 1990). This observation has become well known as Donders' law. Recent papers have reported that Donders' law also applies to other joints with three rotational degrees of freedom, such as for head movements and for movements of the upper arm in the shoulder (Hepp et al. 1992; Hore et al. 1992; Miller et al. 1992; Theeuwen et al. 1993). Just as for the eye, Donders' law is implemented by a reduction in the number of rotational degrees of freedom from three to two: The rotation vectors that describe the position of the head or arm relative to a reference position are contained within a 2-D surface. A quantitative difference that was found between eye, head, and arm movements was that the rotation vectors that describe eye position lie in a flat plane (the so-called Listing's plane), whereas the rotation vectors describing head and arm position lie in a curved surface (Hore et al. 1992; Miller et al. 1992). The implication of a curved surface rather than a flat plane is that the reduction in the number of degrees of freedom from three to two is implemented by the CNS in a different way for the eye and for the arm. For arm movements these results were obtained for pointing movements with the extended arm, which corresponds to rotations in the shoulder joint.

A reduction of the number of rotational degrees of freedom may have large implications for the planning and execution of movements in 3-D. One of the central issues in motor control concerns the kinematic redundancy of human limbs. Human limbs have many joints, some of which have multiple degrees of freedom. Because of the excess of degrees of freedom in these limbs, the position of the end effector can be reached by many joint configurations. When studying single-joint movements or movements of the end effector of a two-joint limb in a 2-D plane, which is what many studies have done, there is a one-to-one relationship between the (set of) joint angle(s) and the target position. However, normal movements are made in 3-D and grasping an object requires as many as six degrees of freedom involving position and orientation of the hand. Because the number of degrees of freedom of the human arm is larger than six, the observation that movements with the same beginning and end points are made in a consistent way with the same joint configurations as a function of time suggests that there is a reduction of the number of degrees of freedom. Therefore the first aim of this study is to study arm movements in 3-D to examine whether they are made in a reproducible way. Moreover, we investigate whether the reduction of the 
number of rotational degrees of freedom in joints, which was studied previously for pointing movements with the extended arm, is also found when the hand is moving in 3 -D to targets in various directions and at various distances relative to the shoulder requiring also flexion/extension in the elbow.

In a recent paper by Soechting et al. (1995) it was questioned whether Donders' law applies to arm movements. Soechting et al. asked subjects to point to targets positioned at various distances and at various directions with respect to the shoulder. It was found that the posture of the arm at a given hand location is not unique, but that it depends on the starting position of a movement. To explain the discrepancy with regard to the validity of Donders' law as reported by previous studies, Soechting et al. suggested that the explanation might be found in the fact that previous studies tested subjects for pointing movements with the extended arm, whereas Soechting et al. tested normal arm movements requiring also flexion/ extension in the elbow. It is indeed not obvious at all that Donders' law is also valid for the hand in normal arm movements. Consider, for example, two cases of a subject pointing with the hand in the same direction: one pointing with a fully extended arm and the other pointing with elbow flexion. In these cases, the upper arm will have different joint configurations. Because the plane that contains the rotation vectors is curved for the upper arm (Hore et al. 1992; Miller et al. 1992), the torsion of the upper arm will be different in these two postures. If the amount of supination/ pronation is the same in both conditions, one might expect a different orientation of the hand. As a consequence, it is not clear whether the orientation of the hand relative to the trunk will be the same for a fully extended arm and for the case in which the hand is pointing in the same direction with arm configurations involving elbow flexion.

The result found by Soechting et al. that the orientation of the hand depends on previous hand positions suggests that there is not a single rotation vector for each position of the hand, but rather that there is some kind of hysteresis in the sense that each hand position may correspond to different rotation vectors for upper arm and forearm depending on previous hand positions. If such were the case, then fitting the rotation vectors by a curved surface, as was done previously by Hore et al. (1992) and Miller et al. (1992), should have revealed a considerable scatter of the rotation vectors relative to the fitted surface. In this context it should be mentioned that several studies have shown that the scatter of the rotation vectors with respect to the fitted surface is larger for the arm, head, and hand (typically $3-4^{\circ}$ ) (Hore et al. 1992; Miller et al. 1992; Theeuwen et al. 1993) than for the eye (typically $<1^{\circ}$ ) (Tweed and Vilis 1987, 1990). On the basis of this observation, Soechting et al. (1995) suggested that the larger scatter may be due to an until recently unnoticed violation of Donders' law for limb movements.

Thus the second aim of this study is to investigate in more detail whether Donders' law is valid for arm movements. In particular we focus on qualitative and quantitative differences between movements of the upper arm and forearm for pointing with the extended arm and for pointing to nearby targets requiring flexion/ extension at the elbow.

The results in this study revealed small deviations from Donders' law. This led us to explore how the violations of
Donders' law are related to the angular velocity vectors. Hysteresis of hand orientation, as reported by Soechting, predicts different arm postures and thereby a different orientation in time for angular velocity vectors for arm movements starting from different positions and directed to the same final position. Moreover, the angular velocity vectors are interesting from another point of view. For the eye with a flat Listing's plane, angular velocities have been reported to correspond to fixed-axis rotations (Tweed and Vilis 1990). Because the rotation vectors for arm movements lie on a curved surface (Hore et al. 1992; Miller et al. 1992; Theeuwen et al. 1993), one might expect that angular velocity vectors are not single-axis rotations, but that the direction of the angular velocity vector changes during the movement to keep the rotation vectors describing arm position during the movement within the curved surface. Therefore the third aim of this study was to investigate the time histories of angular velocity vectors for 3-D movements starting from different positions and aimed at the same final position.

\section{METHODS}

\section{Procedures}

Experiments were performed on 14 adult human subjects. Three of the subjects were familiar with the purpose of the experiment. All subjects gave informed consent to participate in the experiments. Some subjects were tested in different experimental protocols (see Experimental protocol). For protocols 1, 2, and 3, the number of participating subjects was 7, 7, and 6, respectively. The number of subjects who were familiar with the purpose of the experiment was 1,1 , and 3 , respectively. No differences were observed between the results obtained from the subjects who were familiar with the purpose of the experiment and those obtained from the other subjects.

\section{Experimental setup}

Visual stimuli were generated with a quasi-3-D virtual reality system. An HP9000 computer with graphic processor generated video images (frame rate $66 \mathrm{~Hz}$ ) of a 3-D scene. The 3-D scene consisted of a ball $(5 \mathrm{~cm}$ diam) in front of a background having a checkerboard pattern. These video images were projected on a large translucent screen $(2.5 \times 2 \mathrm{~m})$ by a Barco Graphics 400 video projector (red phosphor $\mathrm{p} 56$, green phosphor $\mathrm{p} 53$ ). The subject was sitting on a chair. The position and height of the chair were adjusted such that the two eyes of each subject were positioned $80 \mathrm{~cm}$ in front of the middle of the screen. The position of the chair (and thereby the trunk of the subject) was rotated by $45^{\circ}$ with respect to the screen such that the head of the subject was at a distance of $80 \mathrm{~cm}$ from the screen and such that the right shoulder was at a distance of $\sim 95 \mathrm{~cm}$ from the screen (Fig. 1).

The graphic processor generated a video image of a projection of the 3-D scene on a plane parallel to the projection screen. All video images consisted of two images of the scene, one in green representing the projection of the 3-D scene as viewed by the left eye and one in red representing the projection of the 3-D scene as viewed by the right eye. The subject was wearing a pair of goggles with a red filter (Kodak Wratten number 25) for the right eye and a green filter (Kodak Wratten number 58) for the left eye, providing the subject with stereovision. The balls were presented on a background in the proper perspective relative to the observer such that the background appeared at a distance of $10 \mathrm{~cm}$ behind the screen as seen by the observer. Because the right shoulder of the subject is at a distance of $95 \mathrm{~cm}$ from the screen, the background appears to the subject at a 


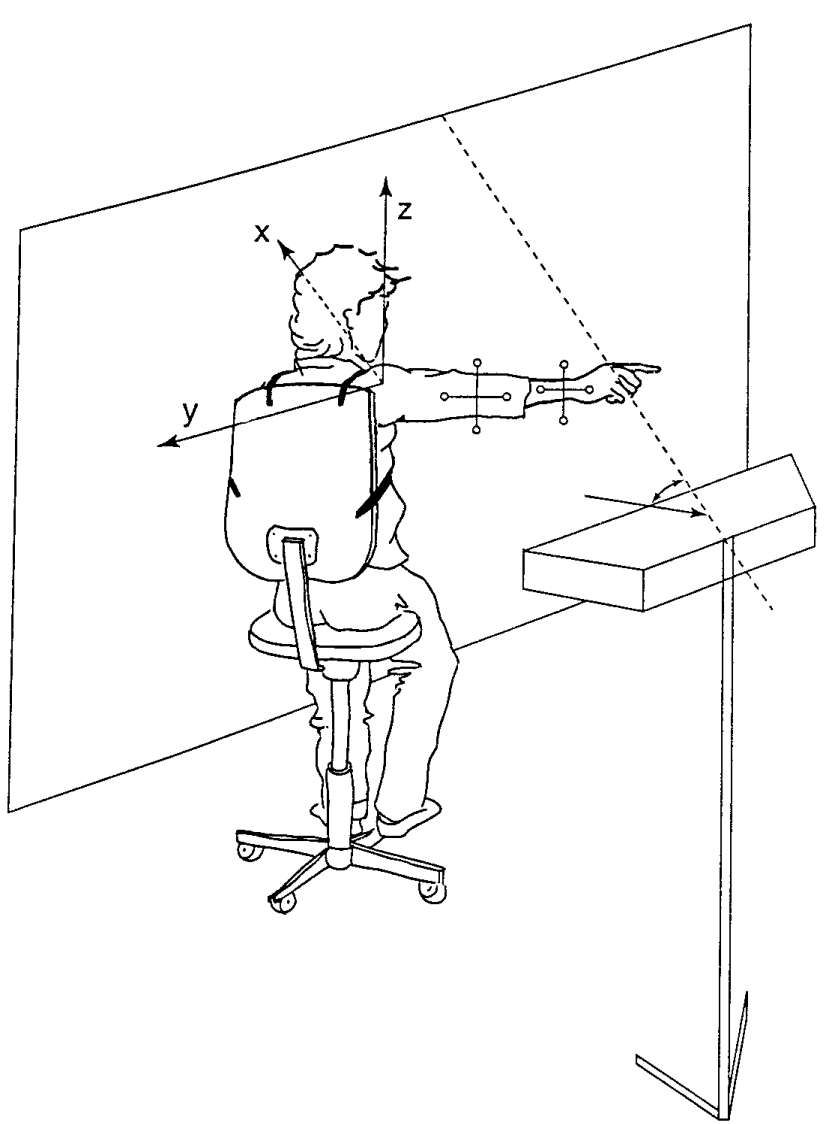

FIG. 1. Schematic view of experimental setup. Subject is sitting behind large screen $(2.5 \times 2 \mathrm{~m})$ on chair that is rotated such that trunk of subject is rotated by $45^{\circ}$ relative to screen. Crosses with infrared-light-emitting diodes (IRED) s on each of 4 arms were attached to forearm and upperarm. OPTOTRAK system is fixated at ceiling at distance of $2 \mathrm{~m}$ behind subject. This OPTOTRAK system is facing downward at an angle of $35^{\circ}$ relative to ceiling. IRED positions are measured in coordinate system that has $Z$-axis pointing upward, $X$-axis pointing orthogonal to (toward) projection screen, and $Y$-axis parallel to screen. Origin of this coordinate system is centered in right shoulder of subject. Usually subject is pointing in direction of screen. However, to clearly show crosses on upper arm and forearm, subject is drawn pointing in slightly different direction.

distance of $105 \mathrm{~cm}$ relative to the shoulder. The balls appeared at various positions relative to the subject.

The position and orientation of the upper arm and forearm were measured with an OPTOTRAK system (Northern Digital), which is capable of measuring the positions of infrared-light-emitting diodes (IREDs ). Crosses with IREDs on each of the four tips were attached to the upper arm just proximal to the elbow joint and at the back of the hand. The lengths of the arms of the crosses were 6 and $12 \mathrm{~cm}$ for the crosses on the hand and upper arm, respectively. The wrist was fixated with a bracelet eliminating any movements at the wrist joint and ensuring that the orientation of the hand and forearm were the same. The bracelet covered most of the hand and also fixated the index finger in full extension such that the forearm, hand, and index finger were all aligned. In addition, subjects had the shoulders strapped to the chair, such that the position of the shoulders was fixed. These precautions were taken to ensure that subjects could make movements in the elbow and shoulder joints only.

The OPTOTRAK system was mounted on the ceiling above the subject at a distance of $2 \mathrm{~m}$ behind the sitting subject. The OPTOTRAK system was facing downward at an angle of $35^{\circ}$ relative to the ceiling such that the IREDs were visible throughout most of the movement range (Fig. 1). The positions of the IREDs are given in a coordinate system centered at the right shoulder of the subject. The $X$-axis is pointing in the direction orthogonal to (toward) the screen. The $Z$-axis is pointing upward and the $Y$-axis is in the horizontal direction parallel to the screen (see Fig. 1). When the orientation of the upper arm and forearm are represented as rotation vectors, these rotation vectors are represented in a different coordinate system, which is explained below in Data analysis.

Although three IREDs would have been sufficient to determine the position and orientation of each arm segment, the fourth IRED on each cross led to an improvement in the accuracy of the position and orientation estimates and allowed the calculations to be made even when occasionally one IRED was not visible by the OPTOTRAK system. When more than one IRED on the crosses was not visible, the data at that point in time were rejected. The position of each IRED was sampled at a frequency of $100 \mathrm{~Hz}$ with a resolution of $\sim 0.1 \mathrm{~mm}$ within a range of $\sim 1.5 \mathrm{~m}^{3}$. In some experiments a sampling rate of $50 \mathrm{~Hz}$ was used. When relevant, this is mentioned in the text. The position of the upper arm and hand was calculated as the average of the positions of the four IREDs attached to the cross. The orientations of the upper arm and forearm were calculated from the orientations of the four IREDs on the crosses in 3-D space ( see Miller et al. 1992). This setup allowed relatively unrestricted movements to be made within most of the natural space.

\section{Experimental protocol}

In the first experimental protocol, subjects were instructed to point to balls that appeared at a distance of $95 \mathrm{~cm}$ from the right shoulder in a plane parallel to the projection screen. These balls had a diameter of $5 \mathrm{~cm}$. The balls appeared in a frontoparallel plane coinciding with the screen at a distance of 0,25 , or $50 \mathrm{~cm}$ from the middle of the screen in eight equally spaced directions (i.e., at angles of $0,45,90,135,180,225,270$, and $315^{\circ}$ relative to the vertical). This gave 17 different target positions ( 8 target directions for distances of 25 and $50 \mathrm{~cm}$ plus the central target, see Fig. $2 A$ ), each of which appeared three times in a randomized order.

In the second experimental protocol, the balls appeared at the same 17 positions mentioned above in three (instead of 1 ) frontoparallel planes at distances of 25,45 , or $65 \mathrm{~cm}$ from the right shoulder (Fig. 2B). In the first trial of this protocol the stimuli were presented in randomized order. In the second trial the stimuli were presented in a sequence such that subjects had to make movements toward or away from the shoulder (i.e., initial and final target positions were in the same direction but at different distances relative to the shoulder). These movements are referred to in the text as "radial" movements. In the third trial the targets were presented at one of the eight different directions but at the same distance relative to the shoulder. In this trial the initial and final targets were either near $(25 \mathrm{~cm})$ or far $(45 \mathrm{~cm})$ from the shoulder. These movements are referred to as "tangential' movements in the text. The duration of these trials varied between 2 and 3 min. All trials were repeated twice.

In the third experimental protocol balls were located at the center and at the four corners of a square (like the " 5 '" on a die) with 30-cm edges (Fig. 2C). The target positions could be positioned either in a horizontal plane at shoulder height, in a frontal plane, or in a sagittal plane. In all cases the central target position was located $50 \mathrm{~cm}$ in front of the shoulder. The targets were positioned such that all edges of the square were either parallel or orthogonal to the projection screen.

\section{Data analysis}

The $X-, Y$-, and Z-coordinates of the four IREDs attached to the crosses were measured by the OPTOTRAK system in a coordinate system that was fixed in space. The position of the cross (including 
A

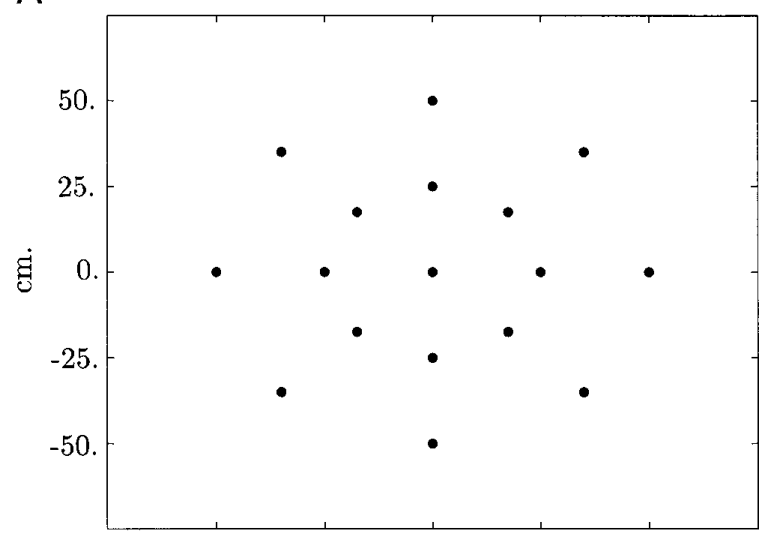

$-50$.

-25 .

cm.

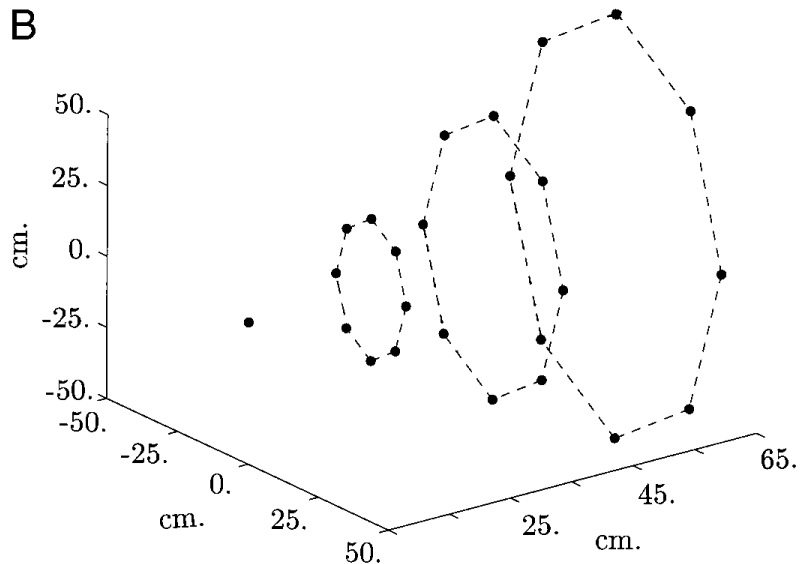

C

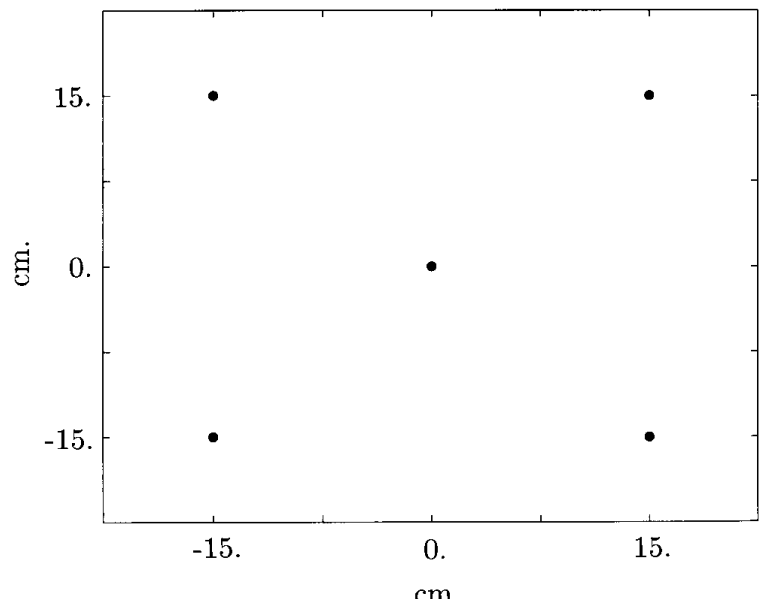

FIG. 2. Schematic overview of stimulus configurations for 3 experimental protocols. For 1st protocol $(A) 17$ targets were presented in vertical plane at a distance of $95 \mathrm{~cm}$ from shoulder. Eight targets are separated over $45^{\circ}$ on circles, each with diameter of 0,25 , or $50 \mathrm{~cm}$ on screen. This gives 17 targets in total. $B$ : side view of balls positioned on circles at a distance of 25,45 , and $65 \mathrm{~cm}$ from right shoulder. Center of right shoulder coincides with filled circle in origin. Each circle has 8 targets separated by $45^{\circ}$. Diameter of largest circle: $50 \mathrm{~cm}$. For 3rd experimental protocol $(C) 5$ targets were presented on screen with distance of $30 \mathrm{~cm}$ between targets on edges of square. Targets were presented at random order in all experimental protocols.

the orientation) was expressed as a 3-D rotation vector, which rotates the cross from an arbitrary reference position (in our case the initial position of the cross at the beginning of the first experimental trial) (see Haustein 1989 for all details).
The procedure to calculate the rotation vectors from the IREDs attached to the cross is described in detail by Haustein (1989) and Miller et al. (1992). These rotation vectors have a direction parallel to the axis of rotation that brings the limb from a reference position to the position of the cross. The magnitude of the vector (which is represented by the values plotted along the vertical axes in Figs. 3 and 4) is equal to the tangent of half the angle of the rotation that brings the limb from the reference position to the cross. For small rotations, the magnitude is approximately equal to the angle in degrees divided by 100 (see Haslwanter 1994). Therefore the units plotted along the vertical axis in Figs. 3, $C$ and $D$, and 4, $C$ and $D$, have to be multiplied by $\sim 100$ to obtain the rotation angle in degrees.

As reported before (Hore et al. 1992; Miller et al. 1992; Straumann et al. 1991), the rotation vectors describing the orientation of the upper arm tend to fall in a curved surface. The curved surface was found by fitting the parameters $a, b, c, d, e$, and $f$ in the second-order function

$$
r_{x}=a+b r_{y}+c r_{z}+d r_{y}^{2}+e r_{y} r_{z}+f r_{z}^{2}+\epsilon
$$

to the rotation vectors $\left(r_{x}, r_{y}, r_{z}\right)^{T}$ (Hore et al. 1992; Tweed and Vilis 1990) such that the residual error $\epsilon$ is as small as possible.

For eye movements a flat plane

$$
r_{x}=a+b r_{y}+c r_{z}+\epsilon
$$

has been fitted usually to the rotation vectors (Tweed and Vilis 1990). When the orientations of the eye are expressed as rotation vectors starting from two different reference positions, $E q .2$ will give two planes with a different orientation. There is one specific reference position, called the primary position, that is orthogonal to the plane with rotation vectors. When this primary position (which is a vector!) is taken as the reference position, the $\hat{X}$-direction in the new coordinate system $(\hat{X}, \hat{Y}, \hat{Z})$ coincides with the primary position. In that case the equation for the plane reduces to $r_{\hat{X}}=0$.

When the rotation vectors are fitted by a curved plane, there does not exist a vector that is orthogonal to all vectors in the curved plane, as for the flat plane in Eq. 2. However, if the coefficients of the quadratic terms $r_{y}^{2}$ and $r_{z}^{2}$ in $E q .1$ are small, fitting a flat plane to the rotation vectors would yield the same values for the coefficients $b$ and $c$ for the flat plane and for the curved plane. Therefore we have varied the reference position in the off-line analysis such that the quadratic terms are minimal. For this reference position, the coefficient $e$ of the cross term $r_{y} r_{z}$ has the largest magnitude. This reference position will be referred to as the "primary position" for the rotation vectors of the arm. To have a measure to quantify how well the surface matched the actual data, the scatter of the data relative to the fitted surface was expressed by the SD (i.e., the square root of the mean of the quadratic distance) of each position vector relative to the fitted surface. In agreement with previous studies, this SD was typically a few degrees.

The coordinate system for showing the rotation vectors for the upper arm and forearm was chosen such that the $X$-direction coincided with the primary position. The $Z$-axis was defined as the unit vector in the direction corresponding to the projection of the (upward-pointing) vertical on the surface with rotation vectors. By this definition, the $Z$-axis is always orthogonal to the $X$-axis. The $Y$-axis then follows from the definition of the $X$ - and $Z$-axis and the convention of a right-handed coordinate system. Notice that the coordinate systems for the rotation vectors of forearm and upper arm will be different, because the surfaces that describe the rotation vectors for the orientation of the forearm and upper arm in general need not be the same.

The angular velocity vectors $\vec{\omega}(t)$ in the shoulder were calculated from the rotation vectors $\vec{r}(t)$ describing the position of the upper arm with the use of the formula 

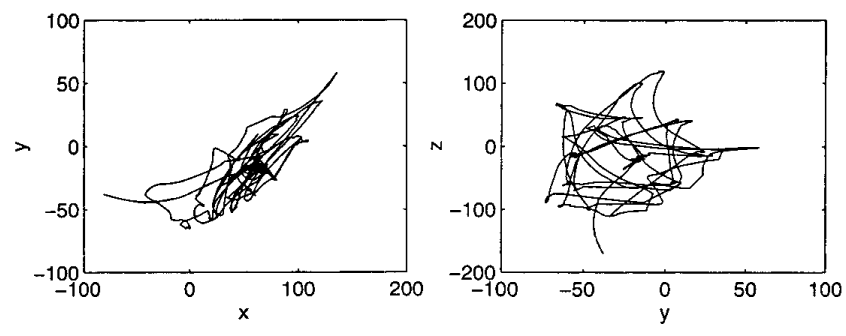

B
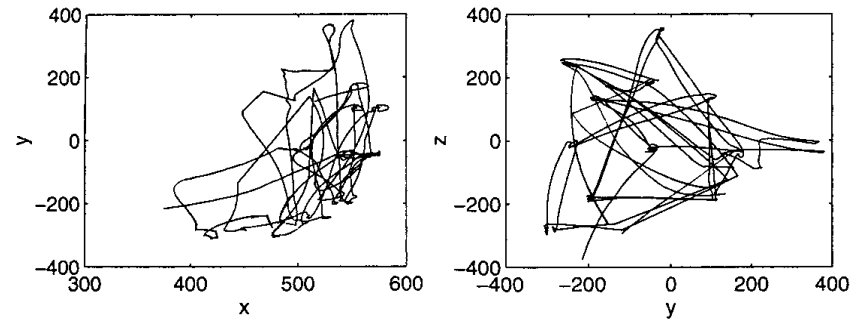

C
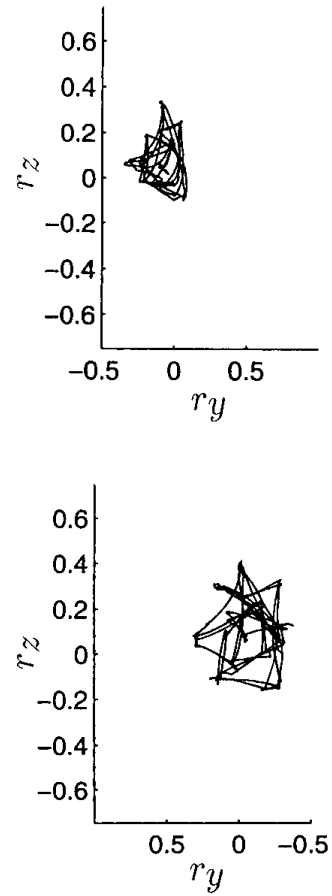

$\mathrm{D}$

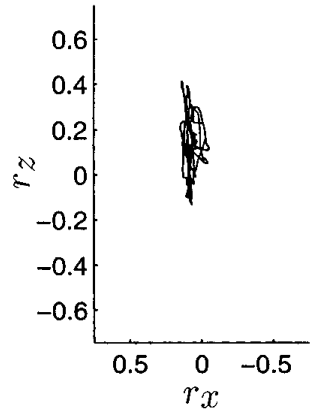

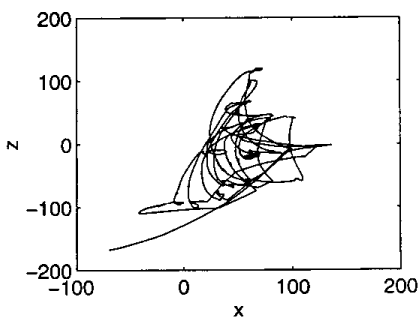
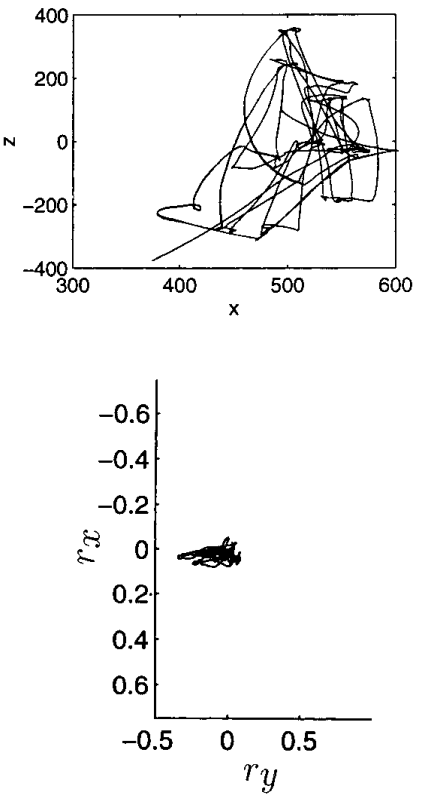

$$
\vec{\omega}(t)=2 \frac{\frac{\mathrm{d} \vec{r}(t)}{\mathrm{d} t}+\vec{r} \times \frac{\mathrm{d} \vec{r}(t)}{\mathrm{d} t}}{1+\|\vec{r}(t)\|^{2}}
$$

(Hepp 1990; van Opstal 1992).

\section{RES ULTS}

\section{Rotation vectors describing the position of upper arm and forearm}

Figure 3 shows the position of the crosses at the upper arm and forearm, respectively (Fig. 3, $A$ and $B$ ), in Cartesian coordinates with the origin at the right shoulder of the subject (Fig. 1), as well as the corresponding rotation vectors (Fig. $3, C$ and $D$ ) representing the orientation of the upper arm and forearm for a subject pointing with the fully extended
FIG. 3. Position and orientation of upper arm and forearm during pointing movements with fully extended arm. $A$ and $B$ : projection of upper arm and forearm, respectively, in $Y-X, Z-Y$, and $Z-X$ planes. Units along axes are in $\mathrm{mm}$. $C$ and $D$ : rotation vectors representing orientation of upper arm and forearm data in $A$ and $B$, respectively, such that front view, side view, and top view on best-fitting plane is provided. Note that $X$-, $Y$-, and $Z$ - coordinates in $A$ and $B$ and those in $C$ and $D$ do not refer to same coordinate system. For rotation vectors $X$-component gives amount of torsion. $Y$ - and $Z$-coordinates correspond to vertical and horizontal rotation vectors in fitted plane, respectively. Units of rotation vectors $(C$ and $D)$ can be converted into deg by multiplication by $\sim 100 \quad$ (see Haustein 1989). Primary position vector, which is normal to best-fitting plane, for data in $C$ and $D$ in world coordinates (see Fig. 1) is given by $(0.83,-0.50,0.24)^{T}$ and $(0.69,-0.63,-0.34)^{T}$, respectively. SD of rotation vectors in $C$ and $D: 2.2$ and $2.5^{\circ}$, respectively.

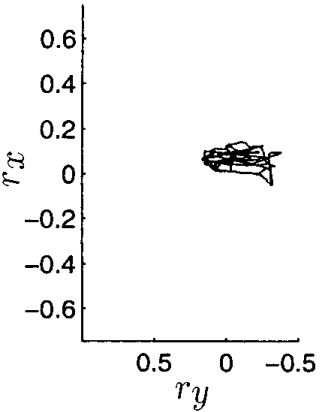

arm to virtual balls at a distance of $95 \mathrm{~cm}$ from the shoulder. The excursion range of the shoulder movements during these pointing movements was $\sim 60 \times 60^{\circ}$. Note that these movements give rise to a larger range of displacements for the cross on the hand than for the cross on the upper arm. The corresponding rotation vectors shown in Fig. $3, C$ and $D$, present a frontal view (left), top view (middle), and side view (right) of the data.

In agreement with previous reports (Hore et al. 1992, 1994; Miller et al. 1992), a flat plane gives only an approximation to the rotation vectors and a significantly better fit is obtained by fitting the rotation vectors by a curved surface. The scatter of the data relative to this curved surface is expressed by the SD of the distance of the rotation vectors relative to the surface. The SD of the data relative to the fitted surface in Fig. 3 is 2.2 and $3.3^{\circ}$ for the upper arm and 

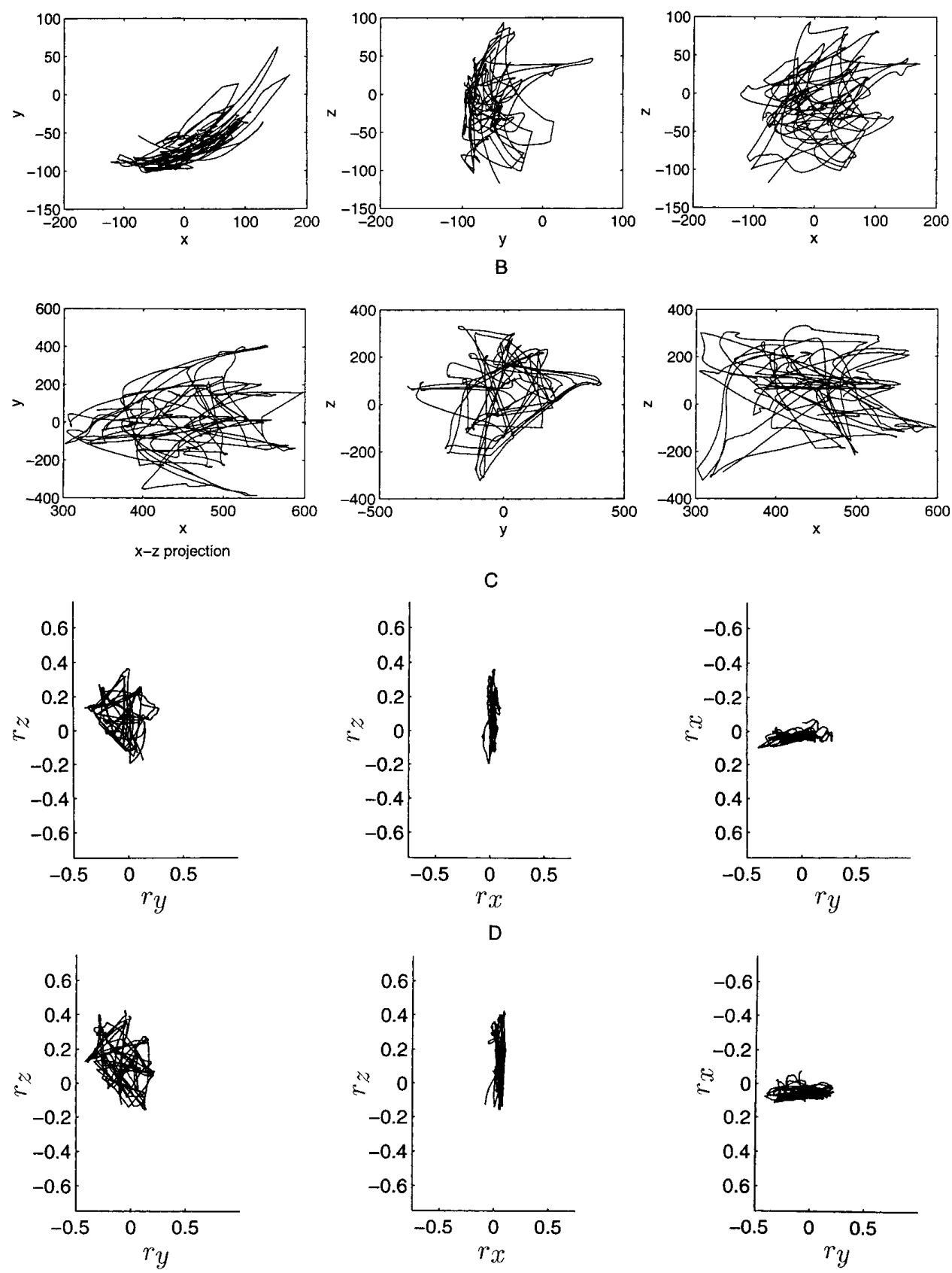

forearm, respectively. The SD for all subjects fell in the range between $1.1-2.3^{\circ}$ and $2.0-3.6^{\circ}$ for the upper arm and forearm, respectively. The mean SD for all subjects was $1.7^{\circ}$ for the upper arm and $2.4^{\circ}$ for the forearm. In an analysis of variance (ANOVA) the SD in data obtained from seven subjects appeared to be significantly larger for the forearm than for the upper arm $[F(1,12)=7.7, P<0.05]$ for pointing with the extended arm.

Figure 4 shows the position of upper arm and forearm (Fig. 4, $A$ and $B$, respectively) and the rotation vectors describing the position of the upper arm and forearm (Fig. 4, $C$ and $D$, respectively) in the same format as in Fig. 3 for a subject who was instructed to touch small balls with the index finger (2nd trial in experimental protocol 2). The data in Figs. 3 and 4 were obtained from the same subject. The
FIG. 4. Position and orientation of upper arm and forearm during pointing movements to targets at distances of 25,45 , and $65 \mathrm{~cm}$ from right shoulder and within range of $60^{\circ}$ along horizontal and vertical directions (see Fig. 2B). Subject was righthanded and all data were obtained from right hand. $A$ and $B$ : projection of position of upper arm and forearm, respectively, in $Y-X, Z-Y$, and $Z-X$ planes. Units along horizontal and vertical axes are in $\mathrm{mm}$. $C$ and $D$ : rotation vectors representing orientation of upper arm and forearm in $A$ and $B$, respectively, such that front view, side view, and top view on best-fitting plane is provided. Note that $X-, Y$-, and $Z$-coordinates in $A$ and $B$ and those in $C$ and $D$ do not refer to same coordinate system. Units of rotation vectors $(C$ and $D)$ can be converted into deg by multiplication by $\sim 100$ (see Haustein 1989). Primary position vector, which is normal to best-fitting plane for data in $C$ and $D$ in world coordinates (see Fig. 1) is given by $(0.86,-0.50$, $0.10)^{T}$ and $(0.66,-0.67,0.33)^{T}$, respectively. SD of rotation vectors in $C$ and $D$ : 2.4 and $3.8^{\circ}$, respectively.

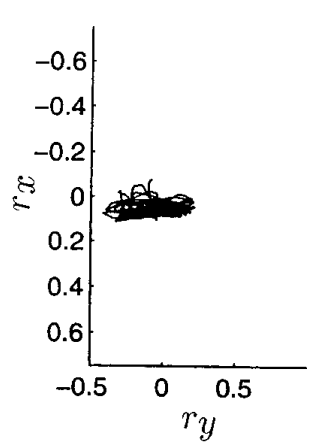

balls appeared at various distances $(25,45$, and $65 \mathrm{~cm})$ and in various directions relative to the shoulder. The instruction to touch the balls requires the subjects to make both shoulder movements and flexion/ extension movements in the elbow. Both for the upper arm and forearm the rotation vectors tend to fall on a 2-D surface. The SD of the data for the upper arm in Fig. $4 C$ relative to the best-fitted surface was $2.4^{\circ}$, which is somewhat larger than that for the data in Fig. $3 C$.

Table 1 shows the SD of the rotation vectors describing the position of the upper arm and forearm for all subjects tested for pointing movements with the extended arm and for movements to targets at different distances along a line passing through the shoulder (radial movements) and to targets in different directions but at the same distance (tangential movements). The rationale for investigating radial 
TABLE 1. SD of rotation vectors

\begin{tabular}{|c|c|c|c|c|c|c|}
\hline Subject & UA & FA & UA & FA & UA & FA \\
\hline SG & 1.7 & 2.1 & 5.5 & 4.4 & 1.7 & 2.7 \\
\hline PS & 2.2 & 2.5 & 2.8 & 4.1 & 2.5 & 3.3 \\
\hline $\mathrm{BB}$ & 1.1 & 2.0 & 2.1 & 5.2 & 2.0 & 4.9 \\
\hline VC & 1.4 & 2.1 & 2.3 & 4.7 & 2.4 & 4.2 \\
\hline Mean \pm SD & $1.7 \pm 0.4$ & $2.5 \pm 0.5$ & $3.2 \pm 1.0$ & $4.4 \pm 0.4$ & $2.2 \pm 0.3$ & $3.6 \pm 0.7$ \\
\hline
\end{tabular}

SD (deg) of the upper arm (UA) and forearm (FA) for pointing with the fully extended arm (columns 2 and 3), for radial movements from targets at $25 \mathrm{~cm}$ from the shoulder to targets at $65 \mathrm{~cm}$ from the shoulder in various directions (columns 4 and 5 ), and for tangential movements to targets at a distance of $45 \mathrm{~cm}$ in various directions relative to the shoulder (columns 6 and 7).

and tangential movements was to investigate the effect of elbow flexion and extension on the orientation of the forearm. Because tangential movements start and stop with the same elbow joint angle, whereas radial movements require different elbow joint angles at the beginning and end of the movement, any effect of elbow flexion would affect the SD of the rotation vectors relative to the fitted plane differently for tangential and radial movements. The results are shown in Table 1, which shows the SD for seven subjects for the upper arm and for the forearm for pointing with the extended arm (columns 2 and 3), for radial movements (columns 4 and 5), and for tangential movements (columns 6 and 7, targets at a distance of $45 \mathrm{~cm}$ from the shoulder).

For the upper arm the mean SD of the rotation vectors relative to the fitted surface was $1.7,3.2$, and $2.2^{\circ}$ for pointing with the extended arm, for radial movements, and for tangential movements, respectively. A one-way ANOVA revealed a significant difference between the SDs of the rotation vectors describing the position of the upper arm for pointing with the fully extended arm and for radial movements $[F(1,12)=10.5, P<0.01]$ and between the SDs of the rotation vectors describing the position of the upper arm for pointing with the fully extended arm and for tangential movements $[F(1,12)=6.34, P<0.05]$. The SD for the rotation vectors describing the position of the upper arm appeared to be not significantly different for radial and tangential movements $[F(1,12)=4.7, P>0.05]$.

With regard to the orientation of the forearm, the same analysis as used to describe the behavior of the upper arm revealed that the rotation vectors describing the position and orientation of the forearm in the "touching" task can also be approximated by a plane (Fig. $4 D$ ). The SD of the data for the forearm in Fig. $4 D$ relative to the fitted 2-D surface is $3.8^{\circ}$. This is slightly larger than the SD of the forearm position data in Fig. $3 D$ for pointing movements $\left(2.5^{\circ}\right)$.

In general, the SD of the data relative to the fitted surface is larger for the forearm than for the upper arm. This is illustrated by the data in Table 1. A one-way ANOVA revealed a significant difference between the SDs for the upper arm and the forearm in the data presented in Table 1 $[F(1,40)=13.56, P<0.005]$. This difference was significant for each of the three conditions tested (pointing with the extended arm, radial movements, and tangential movements). Moreover, the SD for the data describing the position of the forearm for both radial and tangential movements appeared to be significantly larger than the SD for the data describing the position of the forearm for pointing movements with the extended arm $[F(1,12)=58.4, P<0.005$ and $F(1,12)=10.12, P<0.01$, respectively]. The difference between the SDs of the forearm rotation vectors for tangential and radial movements was also significant $[F(1,12)=6.46, P<0.05]$, indicating that the SD is smaller for movements to targets at the same distance relative to the shoulder, which do not require flexion/ extension in the elbow.

For normal movements with flexion and extension in the elbow the surface fitted to the rotation vectors describing the position of the upper arm and forearm in Fig. 4 appears to have almost the same orientation as for the pointing movements with the fully extended arm in Fig. 3. This becomes evident from the fact that the primary direction vector has approximately the same orientation for each of the two conditions, both for the upper arm and for the forearm in Figs. 3 and 4 . The difference in orientation of the surfaces describing the data for the upper arm in Figs. $3 C$ and $4 C$ (defined as the arc-cosine of the inner product between the primary direction vectors to the best-fitted surfaces) was $9.2^{\circ}$. Averaged over all subjects, the mean difference between the orientation of the surfaces describing the position of the upper arm in the pointing task and in the touching condition was $8.3 \pm 5.8^{\circ}$ (mean $\left.\pm \mathrm{SD}\right)$. To determine the variability in the rotation vector data, subjects were tested in repeated trials for the same type of movements. In these repeated trials the orientation of the plane with rotation vectors for the upper arm varied with a SD of $8.9^{\circ}$. This variability indicates that the differences in the orientation of the surfaces fitted to the rotation vectors for pointing movements with the fully extended arm and for movements with flexion/ extension in the elbow were not significant.

For the forearm the difference in orientation for the surface fitted to the rotation vectors in Figs. 3 and 4 was only $4.2^{\circ}$. Averaged over all subjects, the difference in orientation for movements with the fully extended and flexed arm was $10.1 \pm 7.1^{\circ}$. As a measure of the reproducibility of forearm orientation, the orientation was calculated in repeated trials with the use of the same set of stimuli. These repeated trials revealed an $\mathrm{SD}$ of $5.9^{\circ}$ for the forearm.

In summary, these results demonstrate that the rotation vectors that describe the orientation of the forearm and upper arm can be well approximated by a 2-D surface. For both the upper arm and forearm, the orientation of the surface is the same for pointing with the extended arm and for pointing 
to nearby targets, requiring variable amounts of elbow flexion. However, flexion/extension movements in the elbow give rise to a slightly larger scatter of the rotation vectors for the forearm relative to the fitted surface.

\section{Violations of Donders' law}

The amplitude of the scatter of the data along the fitted surface, defined as the SD of the data relative to the surface in Figs. 3 and 4, is typically $\sim 3-4^{\circ}$ (both for the upper arm and hand), which is small considering that the range of torsional shoulder movements is $\sim 180^{\circ}$ and considering the fact that supination/pronation extends this region for the hand to $\sim 360^{\circ}$. This indicates that a thin surface may be a good description for the rotation vectors describing upper arm position during normal pointing and reaching movements. These results can be interpreted as supporting Donders' law because they are consistent with the fact that orientation of the upper arm does not depend on previous arm positions. Yet, a recent paper by Soechting et al. (1995) demonstrates violations of Donders' law. To investigate these contradictory findings, we compared the orientation of forearm and upper arm after movements starting from different initial positions to the same end position.

To test Donders' law, we presented a stimulus configuration with five targets in a frontal plane (see METHODS) with one target on each of the four corners of a square with sides of $30 \mathrm{~cm}$ and with a fifth target in the middle of the frontal square. The distance of the frontal plane containing the five target positions relative to the shoulder was $50 \mathrm{~cm}$.

A one-way ANOVA revealed that there was no effect of initial target position on the position of the hand at the end of the movement to the central target $[F(3,20)=1.3, P>$ $0.1)$. Yet, there was a clear effect of initial target position on the orientation of the upper arm in the middle position. To compare the results for several subjects who have slightly different orientations of the upper arm over the entire range of work space, we calculated the mean torsion of the upper arm (i.e., the amount of rotation along an axis passing through the upper arm) at the central target when coming from target 1 (upper left), 2 (upper right), 3 (lower right), and 4 (lower left), respectively, relative to the mean torsion of the upper arm in the central target position, averaged over movements from all initial four target positions. Torsion is $0^{\circ}$ for the primary position, which is the position corresponding to the position rotation vector orthogonal to the plane fitted to the position rotation vectors. The mean amount of torsion in the middle position for each of the six subjects for movements starting from the four corners of the square to the middle target is shown in Table 2. Columns $2-5$ show the mean difference between the torsion of the upper arm at the central target for movements starting at target 1 (upper left), 2 (upper right), 3 (lower right), and 4 (lower left), respectively, and the torsion of the upper arm in the central target position averaged over all movements from all four starting positions. The mean relative torsion for all subjects and the SD across subjects is shown in Fig. 5. The torsion of the upper arm in the middle position appeared to be slightly but significantly different for different starting positions. For movements starting from the upper left (target 1) to the central target position, the torsion of the upper arm relative to that in the primary position was significantly
TABLE 2. Torsion

\begin{tabular}{lrccc}
\hline \hline \multirow{4}{*}{ Subject } & \multicolumn{4}{c}{ Mean Orientation Upper Arm } \\
\cline { 2 - 5 } & \multicolumn{1}{c}{$\Delta \phi(1)$} & $\Delta \phi(2)$ & $\Delta \phi(3)$ & $\Delta \phi(4)$ \\
\hline ES & $-4.2 \pm 2.0$ & $+3.6 \pm 1.9$ & $+2.7 \pm 2.8$ & $-0.2 \pm 2.0$ \\
SG & $-10.4 \pm 2.0$ & $+9.0 \pm 2.6$ & $+4.6 \pm 6.8$ & $-3.2 \pm 4.7$ \\
GL & $-1.0 \pm 1.6$ & $+0.4 \pm 5.2$ & $+2.0 \pm 1.6$ & $-1.5 \pm 3.6$ \\
CD & $-4.4 \pm 2.7$ & $+1.9 \pm 6.2$ & $+1.4 \pm 1.5$ & $+1.0 \pm 2.4$ \\
EV & $-1.5 \pm 2.5$ & $+0.7 \pm 1.4$ & $-0.2 \pm 1.9$ & $+0.9 \pm 2.7$ \\
BN & $-1.9 \pm 6.3$ & $+3.5 \pm 9.8$ & $-1.5 \pm 2.7$ & $-0.1 \pm 4.7$ \\
Mean \pm SD & $-3.9 \pm 3.4$ & $+3.2 \pm 3.1$ & $+1.5 \pm 2.1$ & $-0.5 \pm 1.5$ \\
\hline
\end{tabular}

Values are means \pm SD. Difference in torsion $(\Delta \phi)$ of the upper arm during pointing to the central target for movements starting from the 4 different starting positions and the torsion of the upper arm averaged over all movements (i.e., for all initial positions) is shown for 6 subjects. The 4 initial target positions are at the corners of a square with edges of $30 \mathrm{~cm}$ in the frontoparallel plane. The target for the end position of the movements is at the center of the square, $50 \mathrm{~cm}$ in front of the right shoulder. Target 1 is at the upper left, target 2 at the upper right, target 3 at the lower right, and target 4 at the lower left of the square.

smaller than that for other starting positions. In an ANOVA the effect of starting position on the torsion of the upper arm in the middle target position appeared to be significant $[F(3,20)=7.65, P<0.0025]$. As reported above, there were no significant differences for the $3-\mathrm{D}$ position of the hand at the middle target position. Nor were there any significant differences for the amplitude or movement time of the movements starting from different initial positions. This indicates that any changes in the amount of torsion of the upper arm at the middle position had to be attributed to the initial starting position of the hand.

\section{Angular velocity vectors}

To investigate whether shoulder rotations during pointing to targets in space are single-axis rotations, as was demonstrated for the eye, we calculated the angular velocity vector at the shoulder during each movement and plotted the angular velocity vector during the movement in 3-D space. If shoulder rotations are single-axis rotations, the angular velocity vector as a function of time should have the same direction throughout an entire movement. Only its amplitude should vary by initially increasing and subsequently decreasing in size.

Figure 6 shows the projection of the angular velocity vectors in the $X-Y, Y-Z$, and $X-Z$ planes for movements from the middle of a square to the upper right and lower left corners of the square. The distance of the initial (central) target from each of the targets at the corners of the square is $21.2 \mathrm{~cm}$. The middle of the square was located at a distance of $50 \mathrm{~cm}$ in front of the right shoulder of the subject. For each target, the trajectories of the angular velocity vectors for four repeated movements have been superimposed.

The large $X$-components of the angular velocity vectors indicate that the angular velocity vectors are tilted out of the $Y-Z$ plane, which is the plane that is fitted to the position rotation vectors. The fact that the angular velocity vectors can be tilted out of the surface, which is fitted to the position rotation vectors, is in agreement with saccadic eye movement data (Tweed and Vilis 1990). The fact that angular velocity vectors are tilted out of the plane with the position 


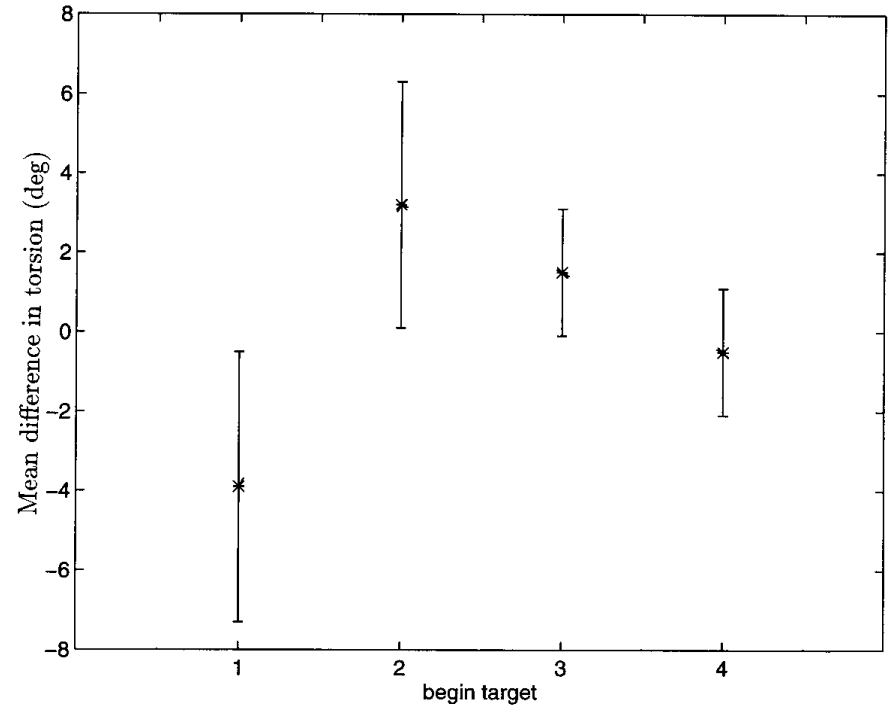

FIG. 5. Mean difference between mean torsion of upper arm for pointing to central target (in deg) for movements starting from target 1 (upper left), 2 (upper right), 3 (lower right), and 4 (lower left) to central target and mean torsion for pointing to central target averaged over movements from all initial positions, averaged over all 6 subjects. Error bars: SD in data across subjects.

rotation vectors was a consistent finding for almost all movements.

Figure 6 also illustrates that the trajectories of the angular velocity vectors during movements in opposite directions are not simply inverted. Clearly, movements to the upper right target position in the frontal plane (data in the 3rd quadrant of the $X-Y$ plane) give rise to angular velocity trajectories that are different from those for movements to the lower left target (see data points in 1st quadrant in the $X$ - $Y$ plane). The projections in the $Y-Z$ and $X-Z$ planes tell the same story. This was a consistent finding for all subjects and was also found for movements in other directions as well.

Another observation, which follows from the data in Fig. 6 , is that the angular velocity data do not fall along a straight line. This means that shoulder rotations are not single-axis rotations, as was reported for saccadic eye movements. The deviations from a straight line are not due to noise in the movement. Any noisy-looking loops in the trajectories are consistent and reproducible for movements with the same initial and final positions (see Figs. 6 and 7). This becomes evident from the fact that the mean correlation coefficient between two angular velocity vectors of movements with the same beginning and end position was $0.89 \pm 0.11$, whereas a linear correlation fitted to the angular velocity vector at 0.01-s time intervals for a single movement was only $0.71 \pm$ 0.11 on the average. This illustrates that the differences between the instantaneous values of the angular velocity vectors during different movements toward the same target are much smaller than the deviations of the instantaneous angular velocity vector from a straight line for each individual movement.

The fact that angular velocities for movements in opposite directions have different trajectories was a common finding for all subjects. We wondered whether this could be due to the fact that movements starting from different targets and directed toward the same central target position were made
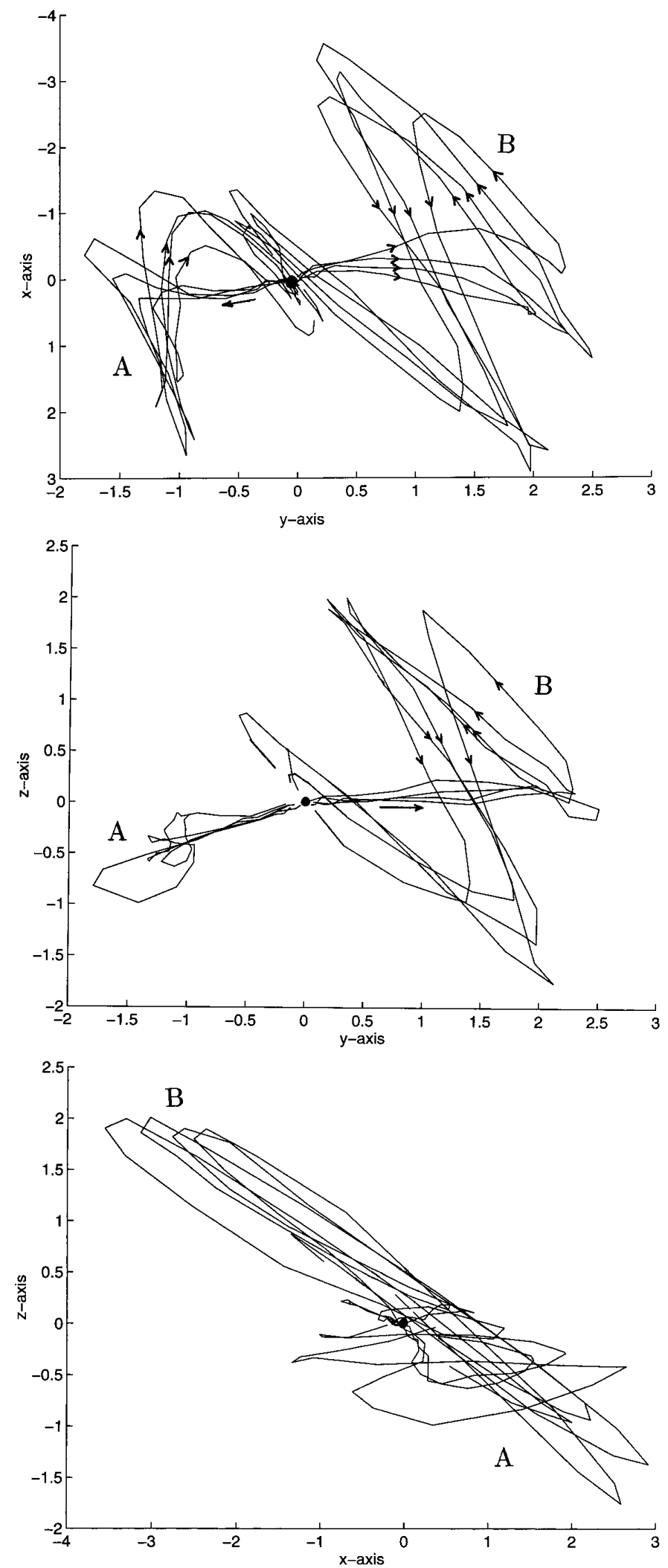

FIG. 6. Projection of trajectory of angular velocity vectors in $X-Y$, $Y-Z$, and $X-Z$ planes for movements between targets in frontal square with $30-\mathrm{cm}$ edges at distance of $50 \mathrm{~cm}$ from right shoulder. Units along axes are in $\mathrm{rad} / \mathrm{s}$. Movements with amplitude of $21.2 \mathrm{~cm}$ started from central position (see Fig. $3 C$ ) and were directed toward upper right (angular velocity vectors labeled A) and lower left (angular velocity vectors labeled B) targets. Units along axes are in rad/s. Mean movement time: $\sim 400 \mathrm{~ms}$. Traces of 4 movements to each target are superimposed. 

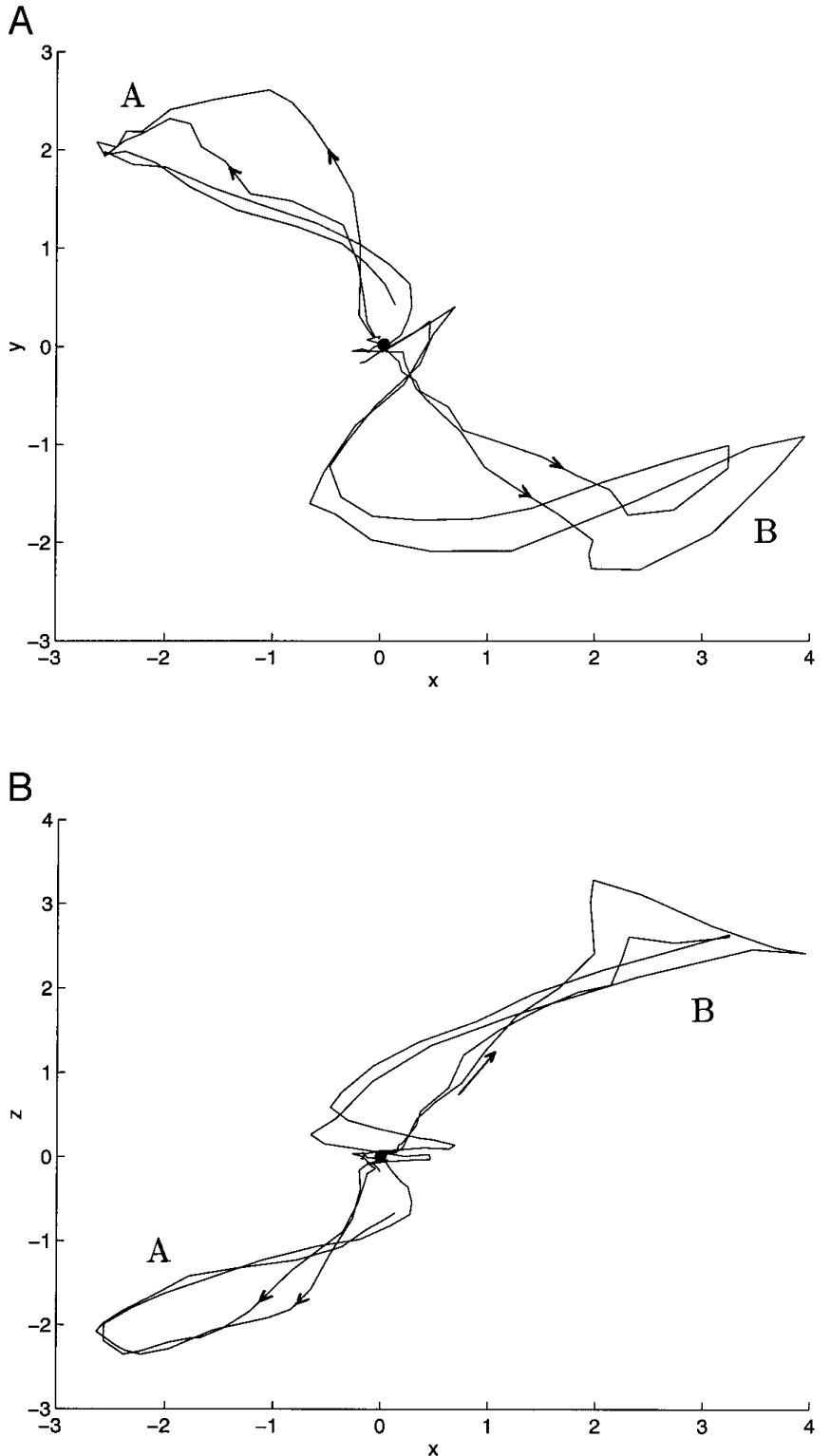

C

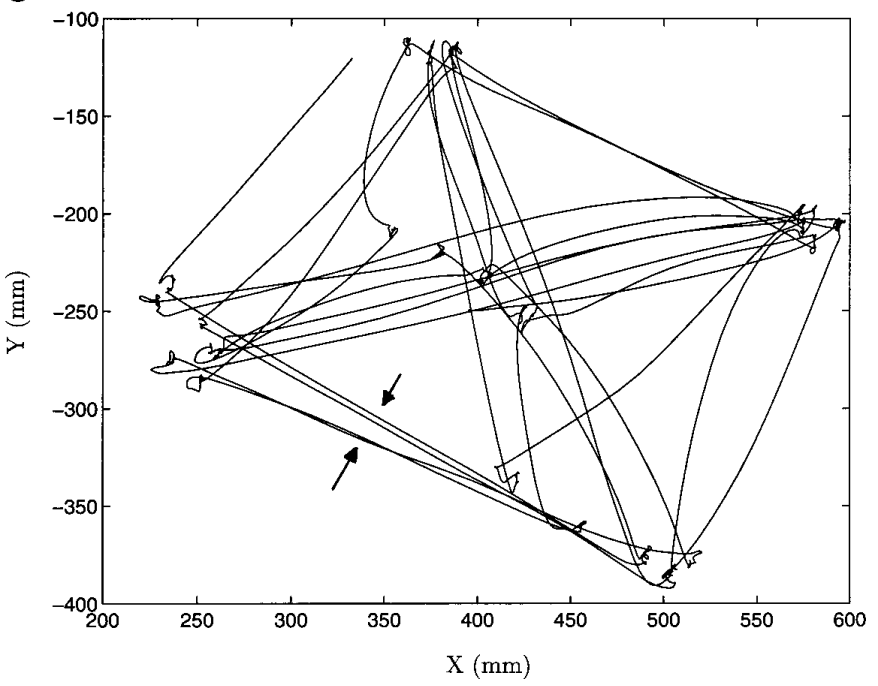

in different parts of work space (the initial position was the same, but the movements were in opposite directions). To investigate the effect of the work space region, we also investigated movements in opposite directions such that the starting position for one movement was the final position for the other one and vice versa. The results are illustrated in Fig. 7, $A$ and $B$, which shows the projection of two angular velocity vectors in the $X-Y$ and $X-Z$ planes for movements in opposite directions (from the lower left target to the upper left target and reversed). As shown before, the trajectories of the angular velocity vectors do not fall along a straight line. In addition, the angular velocity trajectories for movements in opposite directions are not mirror inverted. For the majority of the movements being recorded, the differences between angular velocity trajectories for movements in opposite directions were significantly larger than could be expected on the basis of the variability of the angular velocity trajectories.

Figure $7 C$ shows the corresponding position traces of the cross attached to the upper arm during the movements. Arrows point to pairs of back-and-forth movements. Clearly, the differences between the position traces within each pair of back-and-forth movements are smaller than the differences between the two movements in the same direction. Yet, the angular velocity vectors for movements in the same direction are more alike than the angular velocity vectors of movements in opposite direction (see Fig. 7, $A$ and $B$ ).

\section{DISCUSSION}

The main finding of this study is that the upper arm and forearm violate Donders' law for movements to targets at various positions relative to the shoulder in 3-D space. This result corroborates previous findings by Soechting et al. (1995) and suggests that violations of Donders' law that remained unnoticed in previous studies (Hore et al. 1992; Miller et al. 1992; Straumann et al. 1991) may have been hidden in the scatter of the rotation vector data relative to the fitted surfaces. The fact that previous studies have overlooked violations of Donders' law is not surprising in the light of the result in this study that violations of Donders' law are typically rather small, namely a few degrees. Within this scatter the behavior of the upper arm and forearm during normal arm movements reveals a reduction in the number of degrees of freedom that is similar to that reported earlier for pointing movements with the extended arm. The small violations of Donders' law may well explain why the scatter of the rotation vectors is larger for the arm than for the eye. In the next paragraphs we discuss the results of this study in more detail.

\section{Violations of Donders' law}

The results with respect to the effect of starting position on the orientation of the hand revealed a significant effect

FIG. 7. $A$ and $B$ : projections of angular velocity vectors on $X-Y$ and $X-Z$ planes for movements to targets located within horizontal square at shoulder height with $30-\mathrm{cm}$ edges. Movements were made from lower left to upper left (angular velocity vectors labeled A) and from upper left to lower left targets (angular velocity vectors labeled B). Traces for 2 repeated movements are superimposed. Units along axes are in rad/s. $C$ : arrows point to hand trajectories of 2 pairs of backand-forth movements. 
of the starting position, indicating violations of Donders' law. Deviations from Donders' law have been reported earlier by Tweed and Vilis (1992) in a study in which subjects were asked to make repetitive changes in gaze by combined eye and head movements. These deviations were interpreted as the result of a strategy of the head to decrease the amplitude of repeated movements. Another study that revealed violations from Donders' law was that by Soechting et al. (1995). Their results were compatible with the hypothesis that the final posture minimizes the amount of work that must be done to transport the arm from the starting location. Qualitatively, this hypothesis (minimization of the amount of work to displace a limb) is similar to that proposed by Tweed and Vilis (minimizing the amplitude of movements). However, Tweed and Vilis only noticed this violation of Donders' law for repetitive movements, not for random movements to various targets in space. In our study the violations of Donders' law were small in most test trials, but could be made more explicit for repeated movements to a central target in the middle of four other starting positions on the corners of a square.

The amplitude of this hysteresis effect (up to $10^{\circ}$ ) was smaller than the hysteresis (up to $20^{\circ}$ ) reported by Soechting et al. (1995). The different magnitudes of the effect may be partly due to the range of the movements within the work space, which was $\sim 30 \mathrm{~cm}$ in our study (corresponding to a range of shoulder joint angles of $\sim 30^{\circ}$ ) and $\sim 65^{\circ}$ in the study by Soechting et al. Assuming for simplicity that the effect of hysteresis on the orientation of the hand increases linearly with the amplitude of the movement, the mean difference in orientation of the hand as a function of starting position increases by $\sim 0.1^{\circ}$ per centimeter of movement amplitude (see Table 2), which is close to the mean value that follows from the study by Soechting et al. (1995).

Soechting et al. (1995) investigated several hypotheses to explain the hysteresis. It proved impossible to predict the final posture of the arm purely from kinematics, i.e., on the basis of initial posture of the arm and assuming that Donders' law is obeyed. As mentioned above, one hypothesis was successful in predicting final arm postures, namely assuming that the final posture minimizes the amount of work that must be done to transport the arm from the starting position. However, there may be other explanations as well. For example, Rosenbaum et al. (1995) proposed a model to predict postures of multijoint limbs. In that model, several postures are stored in memory. To make a trajectory the system is thought to weight the stored postures on the basis of spatial accuracy costs (the extent to which the stored postures miss the target) and travel costs (how "expensive" it will be to move to the stored posture from the starting posture). This model clearly predicts final posture dependence on initial posture and therefore predicts deviations from Donders' law. However, a quantitative comparison between theoretical predictions and experimental data has not been done so far. Another explanation for violations of Donders' law may be based on data from Gregory et al. (1987) and Proske et al. (1993), who reported that the discharge of muscle spindles after a ramp stretch of constant amplitude depended on the length history of muscle in the period before the stretch. This tixotropic effect reflects a hysteresis in the discharge of muscle spindles related to the preceding history of muscle length. Because it is well known that muscle spindle re- sponses contribute to the percept of limb position, Gregory et al. (1988) predicted that the hysteresis in spindle discharge would affect position sense in humans. In agreement with this hypothesis, Gregory et al. found that subjects made consistent errors in matching the position of the hand with the other hand. The amplitude of the matching errors depended on the history of the length of the biceps muscle (and thus on positions of the hand) before the matching movement, and the errors were shown to be consistent with the variations in resting discharge of muscle spindles in the cat experiments. It could well be that a similar positiondependent hysteresis of muscle spindle output may have contributed to the fact that the orientation of the hand at the final position, i.e., in the middle of the square, did depend on the starting position of the hand before the movement.

The hysteresis observed in this study may provide an explanation for the fact that the scatter of the rotation vectors relative to the fitted surface is larger for the arm $\left(\sim 4^{\circ}\right)$ than for the eye $\left(\sim 1^{\circ}\right)$. For eye movements, an effect of starting position on the orientation of the eyes at the final position has never been reported and the small variability in orientation of the eye $\left(\sim 1^{\circ}\right)$ has been attributed to some sort of "neural noise." When we assume that the effect of hysteresis on the orientation of the hand at the end of a movement increases linearly with movement amplitude, then a straightforward calculation (see APPENDIX) predicts that the rotation vectors that describe the orientation of the hand will scatter relative to a surface best fitted to the rotation vectors in our data, with an SD of $\sim 3^{\circ}$. If we assume that there is an intrinsic variability (due to neural noise) in the orientation of the hand of $1^{\circ}$, as there is for the eye, then the total scatter can be calculated as the square root of the sum of squares of the two contributions, giving rise to a total $\mathrm{SD}$ of $3.5^{\circ}$, which is well in agreement with the data in this study.

\section{Angular velocity vectors for shoulder movements}

Tweed and Vilis (1990) pointed out that to keep the position rotation vectors (describing the orientation of the eye) in Listing's plane, angular velocity vectors are tilted out of Listing's plane in a specific way depending on the initial eye position. We also found angular velocity vectors that were tilted out of Listing's plane. However, a clear difference was found in the direction of the angular velocity vector during saccadic eye movements and during arm movements. Tweed and Vilis (1990) reported that saccades have nearly fixed rotation axes. We found that the direction of the angular velocity vector during the movement was not fixed but that it varied to a large extent. Moreover, we found that the angular velocity vector for back-and-forth movements was different. There may be several tentative explanations for the complex pattern variations of the angular velocity vectors during the movement.

The first possible explanation follows from the mathematical definition of the angular velocity ( see Eq. 3 in METHODs), which states that angular velocity $\vec{\omega}$ is proportional to $\mathrm{d} \vec{r} / \mathrm{d} t+\mathrm{d} \vec{r} / \mathrm{d} t \times \vec{r}$, where $\vec{r}$ represents the rotation vector describing the orientation. The second component $\mathrm{d} \vec{r} / \mathrm{d} t \times$ $\vec{r}$ is orthogonal to the first component $\mathrm{d} \vec{r} / \mathrm{d} t$ because of the vector cross product. Because the rotation vectors $\vec{r}$ are in a flat plane for the eye, but in a curved plane for the arm, the angular velocity vector $\vec{\omega}$ for the arm must have a more 
complex shape than that for saccadic eye movements. As a consequence, the result in this study that angular velocity vectors for the upper arm are not single-axis rotation vectors may not be surprising. Because the curvature of the surface with rotation vectors is different for different upper arm positions, the term $\mathrm{d} \vec{r} / \mathrm{d} t$ will be different for movements with the same amplitude and direction but with a different starting position. Whether this can explain the different angular velocities for back-and-forth movements quantitatively is not clear and can only be answered after thorough quantitative simulations.

Another possible explanation for the complex shape of the angular velocity vector may be that movements are generated, as is suggested by the equilibrium-point hypothesis, by gradually shifting the hand equilibrium position along a desired trajectory (Flash 1987). By shifting the equilibrium position, which corresponds to the position of the hand in space where the external loads on the hand balance the forces generated at the hand by the muscles, the hand passively follows the equilibrium point. However, because of the stiffness, viscosity, and inertia of the hand, the trajectory of the hand will not be identical to the trajectory of the equilibrium point, because the CNS may not explicitly take into account the inertial and viscous force components when generating appropriate muscle activation patterns. As a consequence, a simple trajectory for the equilibrium point may give rise to complex trajectories for the hand (especially for rapid shifts of the equilibrium point) and therefor, may give rise to complex angular velocities in the shoulder. On the basis of this model Flash (1987) already predicted differences between the trajectories of back-and-forth movements, as observed in the present study. Further quantitative studies are necessary to discriminate between these possible explanations.

\section{Functional implications}

The results of this study show that the rotation vectors describing the position of the upper arm and forearm are contained in a slightly curved sheet with a thickness of a few degrees. The curved surfaces that were fitted to the rotation vectors are shown in Fig. 8. Both surfaces are close to the origin, i.e., passing through the center of rotation in the shoulder. However, to clearly distinguish the surfaces for the forearm and upper arm, the surfaces were shifted. For the upper arm, the curved surface is more or less orthogonal to the upper arm. This means that torsion components in the shoulder (which would become visible as rotation vectors with a significant $X$-component) are rather small. This is not true for the forearm. The surface fitted to the rotation vectors of the forearm is slanted such that the $X$-component is positive (corresponding to supination) for rotation vectors with a positive $Y$-component (i.e., for arm positions to the left). For arm positions to the right corresponding to rotation vectors with a negative $Y$-component, the forearm tends to pronation corresponding to negative components along the $X$-axis. The variations in supination/ pronation are typically $\sim 15^{\circ}$ for movements in a work space of $50^{\circ}$. This is another illustration of the previously reported finding (Theeuwen et al. 1993) that supination/pronation of the forearm varies in a reproducible way as a function of upper arm position during arm movements. The implication is that when a subject starts moving with the palm of the hand pointing downward, the orientation of the palm of the hand will change by $\sim 15^{\circ}$ during pointing in various directions.

Many studies have described the functional implications of Donders' law for eye, arm, and head movements (Hore et al. 1992, 1994; Straumann et al. 1991; Tweed and Vilis 1987). One of the main implications is that it may simplify movement control such that there is no undesired accumulation of torsion after a sequence of movements and such that the amount of torsion is known given the direction of gaze or pointing. However, there are also several important implications for the planning and generation of movements in 3-D.

One of the implications concerns the movement trajectory. When the arm moves from an initial position to a target position, the amount of torsion of the upper arm will change during the movement. When the movement is made with the fully extended arm, any changes in torsion along an axis passing through the upper arm will not affect the trajectory of the hand: it will be on a sphere centered at the shoulder. However, when the arm is flexed at the elbow, changes in torsion during the movement will affect the trajectory of the hand in space. As pointed out by Gielen et al. (1997), Donders' law allows specific predictions about the curvature of the hand trajectory for normal multijoint arm movements. It predicts that most movements of the hand cannot be along straight trajectories. Instead, most movement trajectories have to be curved to satisfy Donders' law.

Recently, De Graaf et al. (1991) have shown that the perceptual space is curved. They reported consistent deviations in an alignment task or in setting the direction of a pointer to a visual target. In a later study De Graaf et al. (1994) demonstrated that the curvature of movement trajectories in slow, goal-directed arm movements is not primarily visually based. However, at about the same time, Wolpert et al. (1994) reported a correlation between the curvature of human reaching movements and the perceptual distortion of curvature, arguing for a contribution of perceptual distortion to the curvature of movements. This conclusion was

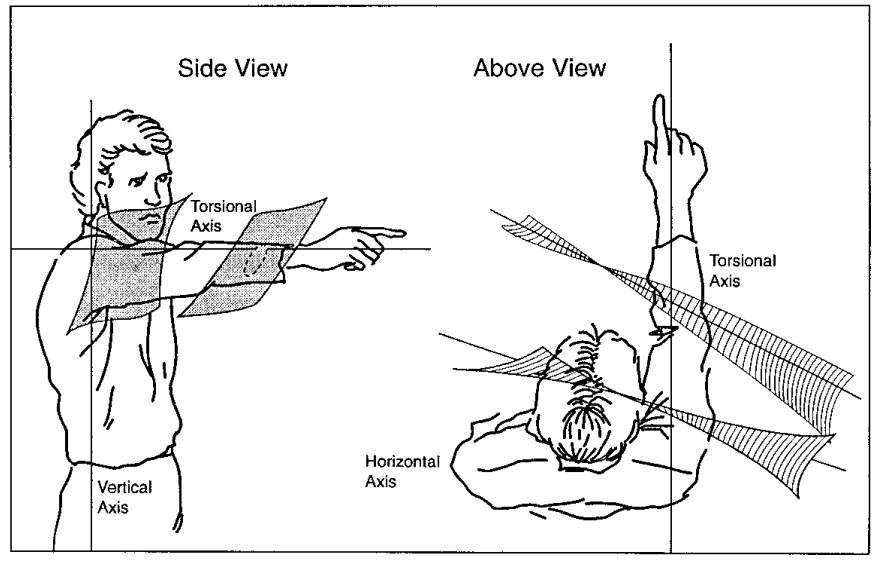

FIG. 8. Schematic drawing of curved surfaces for upper arm and forearm relative to subject. Notice that surfaces should pass through center of shoulder. However, for clarity, surfaces are shifted to indicate whether they represent rotation vectors of forearm or upper arm. Surface corresponding to rotation vectors of upper arm is slightly curved but almost orthogonal to straight-ahead position. Surface with rotation vectors for forearm is tilted along horizontal axis and is in general more curved than plane with rotation vectors for upper arm. 
corroborated by the results of experiments with blind persons and with normal blindfolded subjects (Miall and Haggard 1995), which showed that visual experience influences point-to-point hand movements, leading to a higher curvature for movements made in the frontoparallel plane by sighted subjects due to visual distortions. Moving now from the discussion of the effect of visual perception on movement curvature to eye and limb positioning, it is worthwhile to mention that the hypothesis that visual perception lies at the base of Donders' law is along the lines proposed originally by von Helmholtz (1925). Clearly, quantitative studies are necessary to clarify this issue in detail. In particular, it will be important to decide whether distortions in the visual system impose a curvature of movements, or, the other way around, whether Donders' law imposes curved trajectories that according to theories on the coupling of action and perception may lead to a distorted visual perception. This discussion illustrates that the curvature of movement trajectories may well be the result of several factors. In addition to the factors mentioned above, biomechanical effects or minimization of metabolic energy needed for muscle activation could also affect the nature of movement trajectories.

In the past the shape of movement trajectories (straight or curved) has been used as evidence for movement planning in Cartesian work space (Morasso 1981) or for planning in joint space, respectively (Atkeson and Hollerbach 1985). The idea was that because of the joint rotations, planning in joint space would predict curved trajectories of the hand, whereas straight movement trajectories might suggest planning in work space and the precise coordination of joint rotations to obtain the desired trajectory in space. The discussion in the previous paragraph illustrates that in addition to planning in joint coordinates, several other explanations can be given for the curved movement trajectories that have been observed. For example, when distortions in the mapping from visual space to internal representation of visual space underlie the curved trajectories, the curved nature of movement trajectories is a result of planning in the Cartesian space, which gives a distorted representation of the visual environment, rather than an argument against planning in Cartesian space. However, if Donders' law (which deals with joint rotations) contributes to curved trajectories, then the curved trajectories follow from planning in joint space. These considerations illustrate that the interpretation of the curved nature of movement trajectories, especially when moving from 2-D to 3-D trajectories, is not as straightforward as has been suggested in the literature and would require further studies (Gielen et al. 1997).

\section{A P PENDIX}

The aim of this appendix is to explain how a position-dependent torsion component in the orientation of the upper arm can give rise to a larger SD of position rotation vectors for the arm than for the eye.

The fact that torsion of the upper arm at the end of a movement depends on starting position of the movement will be referred to as hysteresis. For simplicity we will assume that the hysteresis in the torsion of the upper arm increases linearly with distance between starting position and final position of the hand. Firm evidence for this assumption cannot be obtained from our data or from the data by Soechting et al. (1995). However, the data in the study by Soechting et al., which present the hysteresis for initial hand positions in a large range of work space, suggest a larger amount of hysteresis for movements with larger amplitudes.

With this assumption, the error between the mean torsion of the upper arm with the hand in the middle target position and the torsion of the upper arm for the same hand position after a movement starting from an initial position at distance $r$ from the target position at the middle is given by $E=|\alpha r|$, where $\alpha$ is the proportionality factor of hysteresis per unit of distance from the final target position. Averaged over all initial positions in a circular range with radius $R$ in a plane, the error is given by

$$
\begin{aligned}
E & =\sqrt{\frac{\int_{0}^{2 \pi} \mathrm{d} \phi \int_{0}^{R} \mathrm{~d} r(\alpha r)^{2} r}{\int_{0}^{2 \pi} \mathrm{d} \phi \int_{0}^{R} \mathrm{~d} r r}} \\
& =\sqrt{\frac{\pi \alpha^{2} R^{4}}{2 \pi R^{2}}} \\
& =\frac{1}{2} \alpha \sqrt{2} R
\end{aligned}
$$

On the basis of the data in Table 2, which show the differences in torsion at the upper arm for initial positions at the vertices of a square with $30-\mathrm{cm}$ edges, the factor $\alpha$ is $\sim 0.1^{\circ} / \mathrm{cm}$. For initial positions in a circle with radius $R=0.3 \mathrm{~m}$ this gives for the SD $\sigma_{h}$ of the torsion the value $2.1^{\circ}$.

Similar calculations for initial positions in a sphere, rather than a circle, with radius $R=0.3 \mathrm{~m}$ gives an $\mathrm{SD} \sigma_{h}$ of $\sim 3.0^{\circ}$.

Because hysteresis has never been reported for eye movements, we will assume that the $\mathrm{SD} \sigma_{n}$ of eye positions relative to Listing's plane reflects a neural noise component. Then the total SD $\sigma$ relative to the surface with position rotation vectors for arm movements is equal to $\sqrt{\sigma_{n}^{2}+\sigma_{h}^{2}}$. Substitution of the values for $\sigma_{h}$ of 2.1 and 3.0 gives a total SD of 2.4 and 3.2, respectively, which is close to the value of $\sigma$ observed for arm movements (see Table 1).

We are grateful to J. Gielen for assistance during the experiments and to W. Mulder for assistence in data analysis.

This research was supported by the Dutch Foundation for Life Sciences and also in part by a grant to T. Flash awarded by the McDonnell-Pew Foundation for Cognitive Neuroscience.

Address for reprint requests: C.C.A.M. Gielen, Dept. of Medical Physics and Biophysics, University of Nijmegen, Geert Grooteplein Noord 21, NL 6525 EZ Nijmegen, The Netherlands.

Received 6 August 1996; accepted in final form 16 April 1997.

\section{REFERENCES}

AtKeson, C. G. AND Hollerbach, J. M. Kinematic features of unrestrained vertical arm movements. J. Neurosci. 5: 2318-2330, 1985.

De Graaf, J. B., Sittig, A. C., and Denier van der Gon, J. J. Misdirections in slow goal-directed arm movements and pointer-setting tasks. Exp. Brain Res. 84: 434-438, 1991.

De Graaf, J. B., Sittig, A. C., And Denier van der Gon, J. J. Misdirections in slow, goal-directed arm movements are not primarily visually based. Exp. Brain Res. 99: 464-472, 1994.

Donders, F. C. Beitrag zur Lehre von den Bewigungen des menschliches Auges. Hollandischen Beitragen zu den Anatomischen Physiologischen Wissenschaften. Amsterdam: 1847, vol. 1, p. 104-145.

FLASH, T. The control of hand equilibrium trajectories in multi-joint arm movements. Biol. Cybern. 57: 257-274, 1987.

Gielen, C.C.A.M., Vrijenhoek, E. J., and Flash, T. Principles for the control of kinematically redundant limbs. In: Three-Dimensional Kinematic Principles of Eye-, Head-, and Limb Movements, edited by M. Fetter, H. Misslisch, and D. Tweed. Chur, Switzerland: Harwood. In press.

Gregory, J. E., Morgan, D. L., And Proske, U. Changes in size of the stretch reflex of cat and man attributed to aftereffects in muscle spindles. J. Neurophysiol. 58: 628-640, 1987.

Gregory, J. E., Morgan, D. L., And Proske, U. Aftereffects in the responses of cat muscle spindles and errors of limb position sense in man. J. Neurophysiol. 59: 1220-1230, 1988. 
Haslwanter, T. Mathematics of 3-dimensional eye rotations. Vision Res. 35: $1727-1739,1994$

Haustein, W. Considerations of Listing's law and the primary position by means of a matrix description of eye position control. Biol. Cybern. 60 : 411-420, 1989.

von Helmholtz, H. Handbuch der Physiologischen Optik (1st ed.). Hamburg, Germany: Voss, vol. 3, 1867. Third edition translated into English by J.P.C. Southall as Treatise on Physiological Optics. Rochester, NY: Opt. Soc. Am., 1925.

Hepp, K. On Listing's law. Commun. Math. Phys. 132: 285-292, 1990.

Hepp, K., Haslwanter, T., Straumann, D., Hepp-Reymond, M. C., and HENN, V. The control of arm, gaze and head by Listing's law. In: Contro of arm movements in space: neurophysiological and computational approaches, edited by R. Caminiti. Exp. Brain Res. Ser. 22: 307-320, 1992

Hore, J., WatTs, S., AND TweEd, D. Arm position constraints when throwing in three dimensions. J. Neurophysiol. 72: 1171-1180, 1994.

Hore, J., WatTs, S., AND VILIS, T. Constraints on arm position when pointing in three dimensions: Donders' law and the Fick gimbal strategy. J. Neurophysiol. 68: 374-383, 1992.

Miall, R. C. AND Haggard, P. N. The curvature of human arm movements in the absence of visual experience. Exp. Brain Res. 103: 421-428, 1995.

Miller, L. E., Theeuwen, M., And Gielen, C.C.A.M. The control of arm pointing movements in three dimensions. Exp. Brain Res. 90: 415-426, 1992.

Morasso, P. Spatial control of arm movements. Exp. Brain Res. 42: $223-$ 227, 1981.

NAKAYAMA, K. AND BALliet, R. Listing's law, eye position sense and the perception of the vertical. Vision Res. 17: 453-457, 1977.

Proske, U., Morgan, D. L., And Gregory, J. E. Thixotropy in skeletal muscle and in muscle spindles: a review. Prog. Neurobiol. 41: 705-721, 1993.

Rosenbaum, D. A., Loukopoulos, L. D., Meulenbroek, R.G.J., VAughan, J., AND ENGELBRECHT, S. E. Planning reaches by evaluating stored postures. Psychol. Rev. 102: 28-67, 1995.

Soechting, J. F., Buneo, C. A., Herrmann, U., and Flanders, M. Moving effortlessly on three dimensions: does Donders' law apply to arm movements? J. Neurosci. 15: 6271-6280, 1995.

Straumann, D., Haslwanter, T., Hepp-Reymond, M.-C., and HepP, K. Listing's law for eye, head and arm movements and their synergistic control. Exp. Brain Res. 86: 209-215, 1991.

Theeuwen, M., Miller, L. E., And Gielen, C.C.A.M. Are the orientations of the head and arm related during pointing movements. J. Mot. Behav. 25: 242-250, 1993

Tweed, D., Glenn, B., And Vilis, T. Eye-head coordination during large gaze shifts. J. Neurophysiol. 73: 766-779, 1995.

TwEED, D. AND VILIS, T. Implications of rotational kinematics for the oculomotor system in three dimensions. J. Neurophysiol. 58: 832-849, 1987.

TwEED, D. AND VILIS, T. Geometric relations of eye position and velocity vectors during saccades. Vision Res. 30: 111-127, 1990.

TwEED, D. AND VILIS, T. Listing's law for gaze-directing head movements. In: The Head-Neck Sensory Motor System, edited by A. Berthoz, P. P. Vidal, and W. Graf. New York: Oxford Univ. Press, 1992, p. 387-391.

VAN OPSTAL, J. Representation of eye position in three dimensions. In: Multi-Sensory Control of Movement, edited by A. Berthoz. Oxford, UK: Oxford Univ. Press, 1992, p. 27-41.

Wolpert, D. M., Ghahramani, Z., and Jordan, M. I. Perceptual distortion contributes to the curvature of human reaching movements. Exp. Brain Res. 98: 153-156, 1994. 